\title{
CAMBIO TEÓRICO EN LAS FRONTERAS DEL RACIONALISMO CRÍTICO: CONMENSURABILIDAD Y REFUTACIÓN PROGRESIVA EN LAS CIENCIAS SOCIALES
}

Theoretical change in the boundaries of critical rationalism: commensurability and progressive rebuttal in Social Sciences

\section{Cristian Ortega Caro*}

\section{Resumen}

A partir de la epistemología de Karl Popper, se presenta una reflexión que da cuenta de cómo las teorías sociales están sujetas a un especial proceso de cambio epistémico. Para ello se propone un modelo subsuntivo - progresivo, histórico e interpretativo- que edifica, en virtud de relevar el espacio empírico de las teorías, una vuelta a objetivar el desarrollo de las ciencias (aquí, el de las ciencias sociales), y que contiene, además e indirectamente, una crítica a la corriente historicista de la epistemología; todo ello a partir de nuestra propuesta: el Modelo de Refutación Progresiva.

Palabras clave: Racionalismo crítico, cambio teórico, cambio epistémico, falsabilidad, refutación progresiva.

\section{Abstract}

Under the inspiration of Karl Popper's epistemology, we introduce a reflection that brings awereness of how social theories are subject to a particular process of epistemic change. For this reason a substantive model - progressive, historical and intrepetative - is proposed to reveal the empirical space of the theories, an invitation to object the development of the sciences (in particular the social sciences) that contains, in addition and indirectly, a critical view to the historical current of the epistemology; all this from our proposal: the Model of Progressive Refutation.

Key words: Critical rationalism, theoretical change, epistemic change, falsability, progressive refutation.

\section{INTRODUCCIÓN}

Una crítica básica en la evaluación epistemológica de las ciencias sociales es aquélla que señala la subvaloración que posee el criterio de empiriciosidad para conducir el proceso de su desarrollo teórico. A fin de instalar una plataforma para la discusión, signamos tres consideraciones claves: Primero, efectivamente las teorías, a través del tiempo, fenecen. Segundo, y no obstante lo indicado, existe una inagotable generación de nuevas teorías. ${ }^{1} \mathrm{Y}$ tercero, el axioma que sostiene que para la teoría social

\footnotetext{
${ }^{1}$ La ciencia no es la única área del conocimiento que de forma regular renueva sus sistemas conceptuales, teorías y juicios de realidad: el sentido común y los elementos doctrinarios de algunos credos (políticos y religiosos), ciertamente que están en un constante estado de creatividad y transformación. Teorías de la acción social y de la identidad cultural pueden dar cuenta de ello (Giddens, 1997; Berger y Luckman, 2001; Schutz,
} 
no existe un modelo observacional plausible para el análisis del recambio teórico constituye, a lo menos, un juicio que denota una especial deficiencia técnica. ${ }^{2}$ Dicho esto, lo que buscamos es diseñar un sistema —o en su efecto, una cierta lógica- que releve un criterio empírico para configurar dos procesos de desarrollo cognoscitivo: por un lado, un proceso de cambio teórico, orientado en función de transformaciones que le atañen a aquello que denominamos "objeto de conocimiento" y, claro está, de sus sistemas conceptuales asociados $\mathrm{y}$, por el otro, un proceso de cambio epistémico referido a la proliferación e interrelaciones que las estructuras de contenido subsuntivo y los elementos fenoménicos efectúan.

Nuestra propuesta posee una especial inspiración popperiana; ${ }^{3}$ supone, a partir del criterio de falsabilidad, connotar una proyección empírico-interpretativa para el análisis del cambio teórico y guarda, por lo tanto, un cuestionamiento al denominado relativismo epistémico, principalmente a aquellos planteamientos dirigidos al menosprecio por la objetivación teórica y el desgaste de la noción de realidad y conocimiento. ${ }^{4}$

1995). La diferencia con la ciencia es que de ella se espera que su proceso de cambio teórico guarde elementos que, a lo menos, supongan cierta objetividad.

${ }^{2}$ Sin duda que desde la epistemología historicista (que nada tiene que ver con el concepto de historicismo de Popper. Ver nota $\mathrm{N}^{\circ} 8$ ), se podría argüir que el análisis sociológico y lingüístico dirigido a la ciencia, es también, un ejercicio técnico. Sin embargo, aquí hacemos la diferencia entre cuestiones técnicas en un nivel subsuntivo, de aquellas cuyo análisis condicionan la validez del conocimiento a factores sociológicos. Ciertamente que la tesis historicista es plausible para configurar cómo las teorías sociales están sujetas a variables extra-científicas, sin embargo, dicho fenómeno (que en ningún caso negamos) no equivale a sostener que la "veracidad" de una teoría este subdeterminada, tal como lo supone el relativismo epistémico, a condicionantes sociales. A modo de ejemplo, la sociología del conocimiento y la filosofía del lenguaje no son parámetros que resguarden, en principio, criterios de objetividad epistemológica. Para una crítica al relativismo epistémico. Cfr. Sokal y Bricmont, 1999.

${ }^{3}$ Es pertinente aclarar en lo conceptual (y no sólo en lo estratégico) a qué nos referimos con aquello de la inspiración popperiana, aunque de ser honesto, la propuesta es, en general, muy "de Popper". Como sea, nuestro punto de inicio es el de relevar el concepto de refutación y falsabilidad: éstos constituyen el pilar por el cual nuestra propuesta adquiere sentido, y ello en virtud de flexibilizar su operacionalización analítica al momento de dar cuenta del comportamiento de las teorías científico-sociales. Nuestra tesis al respecto es que ellas son, en su confrontación observacional, históricas e interpretativas en el sentido que su testeabilidad es, primero, progresiva (lo que implica una variable temporal) y, segundo, sectorizada (lo que implica una visión analítica). En este punto diferimos con el modelo original, donde la falsabilidad y la refutabilidad poseen consecuencias inmediatas en el criterio de reemplazo y cambio cognoscitivo. Respecto de los límites de esta inspiración, no concurren al debate: el criterio de demarcación —dado que negamos que las teorías sociales pierdan su carácter de cientificidad por el simple hecho de la irrefutabilidad - la tesis del indeterminismo, la crítica al historicismo y la crítica a la inducción (temáticas que, por razones obvias, no son parte de este artículo).

${ }^{4}$ No obstante estas observaciones, no se pretende desarrollar aquí una crítica sistemática al relativismo epistémico (no es nuestra intención, por ahora), sino sólo la de reformular un criterio de cambio epistémico para el análisis de las teorías sociales que guarde componentes de objetivación empírica. Sin embargo, este simple hecho funda un cierto nihilismo en contra de las afirmaciones relativistas: el fin de la verdad (o su intento por buscarla) y de la objetivación (o el fin de la actitud que la sustenta). Como sea, el modelo aquí propuesto, creemos, supone un espectro para una crítica al ejercicio relativista. 
No está demás mencionarlo, todo esto es muy hipotético y forma parte de un proyecto mucho más diverso.

A efecto de dilucidar algunas cuestiones conceptuales, a continuación detallamos la analítica que conforma el proceso de desarrollo teórico aquí propuesto: $:^{5}$ la noción de Cambio Teórico, en un cuadro de dos teorías rivales, implicará, simplemente, el traspaso desde una teoría $Z$ a una teoría $Q$; ello, claro está, en un nivel muy material de constatación. Si este simple traspaso-fin-emergencia constituye un avance en el conocimiento, esto es, si los contenidos tanto empíricos como teóricos adquieren una mayor pertinencia respecto de la realidad que pretende ilustrar, ${ }^{6}$ hablaremos, además, de inflexión cognoscitiva; situación que, evidentemente, será explícita en la investigación práctica y sobre la cual será dable, a nivel de conclusiones epistemológicas, señalar dichos cambios. Por el contrario, si la nueva teoría es una reformulación estética, formal o nominal, cuya variación constituye un proceso intrascendente respecto de la extensión de información empírica o del descubrimiento de nuevos fenómenos, hechos o procesos, en dichos casos sólo hablaremos de un cambio teórico, cuestión circunscrita más a una variación en el ámbito del lenguaje que en el plano de la información empírica. Asimismo, si se presentasen relecturas teóricas (nuevas interpretaciones o reeclecticismos) que establecen nuevas explicaciones o interpretaciones para nuevos hechos sin que éstas tengan relación con un proceso inflexivo ante una teoría rival, en estos casos también hablaremos de un cambio cognoscitivo, pero no de un cambio teórico (tal como ocurre, por ejemplo, con la inclusión de hipótesis ad hoc, donde se presenta un avance cognoscitivo e informativo pero no un cambio formal a nivel de la teoría). En consecuencia, la inflexión epistémica implicará la concurrencia de ambos procesos: a nivel de la estructura formal de las teorías - la teoría Z efectivamente habrá de reemplazar a una teoría $Q-\mathrm{y}$, por otra parte, la nueva teoría deberá, necesariamente, contener nuevos contenidos empíricos que serán más completos y/o pertinentes que los de la teoría anterior. Con todo, los cambios a nivel cognoscitivo hacen mención, principalmente, a las leyes de subsunción y la estructura de verdad que poseen las teorías: explicaciones, interpretaciones, generalizaciones descriptivas e, incluso, inferencias estadísticas que se puedan desarrollar a partir de la investigación aplicada. En síntesis la inflexión cognoscitiva se refiere principalmente al

\footnotetext{
${ }^{5}$ El modelo hipotético-deductivo, si bien no es precisamente un diseño destinado a dar cuenta del fenómeno particular del cambio teórico, constituye un buen ejercicio por racionalizar el progreso del conocimiento científico y, claro está, de la sociedad y la historia. En efecto, hemos optado por la noción de cambio, toda vez que posee una impronta con menos carga valórica que la de progreso y constituye, por cierto, una actualización conceptual que Popper, dado su optimismo, no pudo ver.

${ }^{6}$ Existe en esto un evidente riesgo relativista, dado que se podría dudar respecto de los criterios que determinan la pertinencia o no pertinencia de una teoría. Desde ya, convenimos en que dicha decisión nada tiene que ver con factores sociales o históricos. Por el contrario, suponemos que el valor que guarda una teoría depende de la capacidad interpretativa que logra generar a partir de su entramado empírico.
} 
descubrimiento (o creación) de conocimiento empírico. La inflexión teórica se refiere a los cambios que se suscitan en la estructura técnico-formal de una teoría. La inflexión epistémica es la simetría y coherencia de ambos procesos, esto es, el equilibrio entre la extensión del horizonte empírico y su sistematización en una estructura explicativa o interpretativa. $^{7}$

\section{¿ESTÁ INDETERMINADO EL CAMBIO TEÓRICO EN LA TEORÍA SOCIAL?}

Un factor clave que guía este trabajo, dice relación con cuestionar la afirmación historicista que supone la inexistencia de elementos empíricos, observacionales o lógicos que determinan el fin de una teoría y la emergencia de una posterior. ${ }^{8}$ Pongamos por caso el siguiente ejemplo: la Teoría de la Dependencia es un modelo que, desde la perspectiva historicista, hubiese sido poco pertinente a la elucidación de la crisis económica y financiera acaecida en el primer semestre del año 2009: Si estos imaginarios dependentistas hubiesen diseñado nuevas interrogantes, identificado nuevos hechos y elaborado sistemas de hipótesis o marcos explicativos alternativos, difícilmente la economía convencional hubiese estado dispuesta a re-conceptualizar la crisis a partir de, por ejemplo, el desequilibrio entre centro-periferia (economías de bloque y economías marginales) o en función de los desajustes que genera "el modelo" a economías tercermundistas, dependientes y monoproductoras. Aunque hubiese existido cierta asertividad en dichos juicios, pocos estarían dispuestos a citarla, so pretexto de anacronismo discursivo. Por el contrario, el paradigma centró su análisis en

\footnotetext{
7 "Las teorías son redes que lanzamos para apresar aquello llamamos "el mundo": para racionalizarlo y dominarlo. Y tratamos que la malla sea cada vez más fina” (Popper, 2004:57). Suponemos que teorías generales, de alcance medio, micro-teorías, contextuales, interpretativas, causal-mecanicistas, sea lo que sea, no escapan a esta simple, pero asertiva definición.

${ }^{8}$ En la reflexión epistemológica clásica (Newton-Smith, 1987; Kuhn, 1996) existen dos Modelos referidos al Cambio Teórico: uno es el Modelo Racionalista (o Internista) — que identifica a Poppery el otro es el Modelo Historicista (o no racionalista o externalista) que se edificó en función de Kuhn y Feyerabend. El primero supone que el cambio teórico se concreta a partir de desajustes internos que acaecen en la base empírica de la teoría. Un nuevo hecho - imprevisible desde la lógica interna, pero efectivo desde una óptica realista - implicará, necesariamente, transformaciones en la base fundacional de la teoría; cambia, por lo tanto, la estructura conceptual y significacional, los criterios de verdad y objetividad, las relaciones lógicas entre los hechos y el armazón técnico de la teoría. Por el contrario, en el Modelo Historicista el cambio teórico se produce a partir del condicionamiento que la estructura espacio-temporal ejerce sobre la producción del conocimiento. En principio posee dos variantes: a) en virtud de los acuerdos que se generan al interior de la comunidad científica (por decisión paradigmática, arbitrariedades, caprichos o por el ejercicio de poder que ejercen elites políticas, económicas y morales). b) los cambios se generan bajo la regla general del determinismo producido por circunstancias, fenómenos o hechos de carácter histórico y/o cultural, los cuales pueden ser perfectamente independientes a la actividad interna que desarrolla la comunidad científica. Dos alcances: a) nótese que el concepto de Historicista utilizado aquí, nada tiene que ver con el concepto de Historicismo utilizado por Popper en La Miseria de Historicismo (1973); b) Imre Lakatos (1993 y 2002) identifica y diferencia entre Historia Externa e Interna de la Ciencia. Ciertamente, que el acento para definir las corrientes señaladas deben su conceptualización a lo signado por este último.
} 
factores legislativos, especulación bursátil e ineficiencia del sistema bancario y financiero, pero no en elementos referidos a la estructura macro-económica mundial. Desde una epistemología historicista, bien se podría argüir que la teoría de la dependencia no posee correspondencia con la realidad que pretende interpretar: su sistema teórico y observacional, su sistema de enunciados, el explanans y en general, el modelo explicativo que podría construir, sería extemporáneo respecto de la tendencia actual.

Sin embargo, desde la perspectiva de la contrastación empírica, muchas de las teorías previas a la crítica postmoderna no tendrían porqué estar excluidas de la moda intelectual. En principio, desde la crítica interna, no existen contraejemplos empíricos efectivos ni lógicos que impidan la vigencia de ésta u otras teorías. Relegar estos modelos sólo respondería, tal como lo señalara Popper, a un interesado acto convencionalista (1994:61-62; 2004:75-78), en virtud de las presiones que ejerce algún grupo o sector social.

Desde la otra orilla, pero bajo la misma lógica, el Racionalismo Crítico es claro respecto de evidenciar cómo algunas teorías sociales escapan a la crítica empírica. Tomemos como ejemplo la Teoría de la Identidad: la lógica de la refutabilidad de Popper arguye que modelos teóricos ultra explicativos o interpretativos — que todo lo explican bajo su sistema de enunciados- no sólo son signados de sospecha mitologicista (1994:59), sino que además rehúyen de la falsabilidad y el criterio de cientificidad que ello implica. En efecto, así como la Teoría de la Dependencia fue eliminada vía convencionalismo, bajo la misma lógica la Teoría de la Identidad posee plena vigencia: todo es identidad: cultural, territorial, social, étnica, religiosa, linguística, nacional, continental, etc., cualquier cosa es dable de ser interpretada bajo dicho patrón, que en lo formal disfraza, fragilidad explicativa por una supuesta capacidad deductiva.

En consecuencia, bien podemos plantear que en las ciencias sociales no existe una matriz (un formato o una reflexión) que nos conduzca hacia la formulación de una estructura racional y plausible que visualice, desde componentes empíricos, el cambio teórico e inflexión epistémica; por el contrario, presenciamos una sucesión y superposición de modelos teóricos que actúan unos sobre otros, de forma paralela y bajo una constante tensión. Pareciera existir una cierta ambigüedad al momento de identificar un criterio objetivo de inflexión, lo que no debiera implicar la inexistencia de algún criterio empírico de cambio epistémico. Sostenemos que, primero, dicho criterio está oculto y funciona subrepticiamente y segundo, que ha sido transfigurado bajo un erróneo modelo de interpretación histórica. Por lo tanto, y dada la 
inestabilidad empírica ${ }^{9}$ de las teorías sociales, postulamos que el desarrollo epistémico se debe a un particular proceso de refutación progresiva; evidentemente, mucho más flexible que los criterios propuestos por el primigenio Racionalismo Crítico. ${ }^{10}$

Por el contrario, y no obstante los argumentos provenientes de la epistemología historicista, la inflexión epistémica - bajo un criterio progresivo- requiere de un criterio de conmensurabilidad: las teorías sociales si bien pueden pertenecer a corrientes filosóficas totalmente opuestas existe entre ellas un cierto grado de familiaridad que intuye cierto dialogo. Independientemente de las diferencias espacio-temporales desde donde proliferan las teorías, éstas no varían en el objeto de estudio sobre el cual dirigen sus análisis. En rigor, para la construcción del objeto se utilizan los mismos explanans, los mismos compendios filosóficos, los mismos sistemas conceptuales —en su efecto, el mismo sistema lingüístico- y, ciertamente, los mismos sistemas metodológicos. Pese a las diferencias que cada corriente pueda hacer de la base meta-teórica disponible, y claro está, de la traducción teórica de la información empírica disponible —que bajo la posición de Feyerabend impediría aplicar criterios de conmensurabilidad entre teorías rivales (1984:106-119) — desde nuestra perspectiva ello no niega la reflexión en torno a la comparabilidad y el desarrollo teórico. Si bien los conceptos y sus significantes son extrapolados de un modelo a otro, existe una matriz argumental base; un metaexplanans que inspira en términos muy generales la construcción objetual del mundo que, pese a responder a requerimientos metodológicos diferenciados, no supone grandes discrepancias conceptuales entre una y otra corriente. Sin importar que el objeto de estudio sea la cultura, el sistema social, la clase, el sujeto, el actor o la acción; e independiente que la perspectiva sea del tipo macro o micro social, o que nuestra base argumental sea objetivista o subjetivista o que la matriz filosófica o metaexplanans no nos conduzca, en última instancia, hacia contradicciones taxonómicas o interpretativas gravitantes, las teorías sociales poseen un espíritu - lingüístico, en última instanciaque las hace participe del mismo destino. Desde luego, no descartamos que, tal vez, sea dable consignar algún tipo de diferencias de sentido o de grado significacional respecto de la interpretación de los conceptos utilizados o de los énfasis que proponen las metodologías, pero ello no debiese implicar posturas imperativas de

\footnotetext{
${ }^{9}$ Por el contrario, y muy conjeturalmente, la estabilidad de las teorías sociales radica en el acto interpretativo que es dable aplicar al explanans, situación que viene a compensar la debilidad epistémica que produce la incapacidad estructurante que genera la ambigüedad subsuntiva.

${ }^{10}$ El Racionalismo Crítico, a través de la metodología de la falsabilidad determina que las hipótesis científicas - en su efecto las conjeturas que un investigador genera para dar respuestas a problemáticas de interésnecesariamente deben ser expuestas a una prueba empírica; si ella predice lo que conjeturaba, dicha hipótesis se da por corroborada; si no es así, la hipótesis se da por falseada. Cuando esto último ocurre se puede hablar de progreso científico y ello en razón de que la falsación impone, más allá de explicitar el error, la necesidad científica de formular nuevas hipótesis y, por lo tanto, de generar nuevas conjeturas que promuevan el avance del conocimiento.
} 
inconmensurabilidad. En efecto, las incompatibilidades teóricas, conceptuales y metodológicas entre uno y otro modelo, pertenecientes o no a una misma corriente teórica, se generan, por una parte, al nivel del contacto subsuntivo que los términos teóricos entablan con la realidad empírica y por el otro, de acuerdo a las características particulares de cada escenario histórico sobre el cual el proceso técnico de la elaboración teórica adquiere proyección investigativa. Sin embargo, no obstante la libertad interpretativa que es aplicable a la utilización de algún explanans, aquí se sostiene que dicha contextualización no amerita la aplicabilidad de algún criterio de inconmensurabilidad, ni la negación de un proceso inflexivo a nivel de la información empírica, por cuanto la matriz metateórica guardaría cierta univocidad que la hace incólume al contexto sociocultural de donde emerge la nueva teoría y de la información empírica que se utilice para su elaboración.

\section{MODELO DE INTERPRETACIÓN EMPÍRICA PARA EL CAMBIO EPISTÉMICO ${ }^{11}$}

El Modelo propuesto se compone de tres subsistemas:

a) Descubrimiento de desajustes empíricos.

b) Refutación Progresiva.

c) Proceso de proliferación significacional y conformación de nuevas teorías.

A. Descubrimientos de desajustes empíricos. La visión tradicional respecto del descubrimiento de contraejemplos empíricos en una teoría dice relación con el proceso de refutación que es susceptible de ser aplicado. Desde la óptica de Popper, las teorías se componen de una serie de presupuestos epistémicos tanto teóricos como observacionales que se estructuran indistintamente en un sistema conceptual, en un sistema de enunciados y en un sistema de hipótesis. En Conjeturas y Refutaciones (1994) y, por cierto, en La Lógica de la Investigación Científica (2004), Popper sostiene que las teorías se componen de una estructura de información o en un sistema de conjeturas que están sujetas a una constante contrastación empírica, o en su efecto, sujetas a la metodología de la falsabilidad. Si el proceso de falsación es exitoso, la teoría será refutada; de no ser así, se da por corroborada y puede, en principio, seguir funcionando como un referente explicativo o interpretativo. Ésta es,

\footnotetext{
${ }^{11}$ La base de esta estructura supone, en principio, dos consideraciones clave: Existen teorías sociales que construyen su mundo objetual a partir de un planteamiento micro-teórico, esto es, a partir de los componentes subjetivos que involucra el fenómeno del sujeto o actor social y, evidentemente, de todo un entramado de símbolos y significados. Asimismo, existen teorías cuyo planteamiento es macro-teórico donde la conformación objetual se encuentra en algún tipo de estructura social, sea ésta histórica, económica o cultural (o cualquier cosa) donde la definición del sujeto o actor se subordina a la definición de las tendencias generales que posee un hecho o fenómeno estructurante. Sin detallar las especificidades técnicas que cada perspectiva contiene - y de las síntesis que en la investigación práctica se efectúa - la proliferación de teorías, es desde nuestra óptica un proceso eminentemente subsuntivo, que solo difiere en grados de contenido informativo, según los enfoques teóricos utilizados, las metodologías y las intenciones científicas y laicas que las investigaciones y los científicos se proponen.
} 
desde el racionalismo crítico, la formula por la cual el desarrollo de las ciencias naturales ha operado hasta ahora. Sin embargo, en las teorías sociales, el proceso de refutación no posee una aplicabilidad tan milimétricamente racional; sólo mediante un acto forzado, el desarrollo de las teorías sociales podría ser interpretado bajo el criterio de falsación. Por otra parte y dado el fundamento empírico de las ciencias sociales, el proceso de refutabilidad porque tener complicaciones en su aplicabilidad; existe, en la construcción del objeto como en el diseño del discurso teórico, un equilibrio plausible entre elementos de hecho, elementos conceptuales y enunciativos: en ello las teorías sociales guardan un principio lógico similar al de otras teorías, lo que no significa, tal como lo pretendía el Positivismo, homologar ciencias sociales con ciencias naturales. En este sentido, el sustrato empírico funciona como un elemento que otorga el origen epistémico al objeto, orienta su construcción conceptual, pero no define su estructura teórica. Muy en el estilo de Popper, la reconstrucción teórica se determina más por la injerencia que posee el acto imaginativo y por la capacidad de elucubración teórica del científico, que por la simple identificación de los hechos en la realidad empírica (1994-2004).

Visto este escenario, el primer paso en el proceso de inflexión epistémica en la teoría social, es que éstas no funcionan como lo hacen, en general, las ciencias naturales, esto es, un proceso metodológico que, en el cuadro de dos teorías rivales, conduce necesariamente a la eliminación de una de las dos (o más) teorías. Por el contrario, establecemos que el proceso subsuntivo en la teoría social y, en particular, respecto de las condiciones que cimentan una crítica interna —en el contexto de la proliferación o descubrimiento de un nuevo hecho o contraejemplo empírico- opera subrepticiamente, lo que significa que su poder de refutación no posee, en primer lugar, la trascendencia epistémica que en las ciencias naturales si posee; como tampoco determina un proceso claro de eliminación teórica. Podemos afirmar que, a la larga, los elementos subsuntivos y los contraejemplos empíricos funcionan, pero éste es un proceso gradual y soterrado. Sin duda que cuestiones vinculadas a perspectiva epistémica y vigencia explicativa se ven alteradas, pero dicho proceso no es ni directo ni instantáneo. Presuponemos que todas las teorías sociales presentan, en algún momento de su vigencia epistémica, algún grado de incoherencia empírica entre sus presupuestos teóricos, el sistema de hipótesis y la realidad empírica que pretenden interpretar; sin entrar en detalles respecto del origen de estas incoherencias (que, en términos generales, podrían deberse a deficiencias de operacionalización interpretativa) presupuestamos que ello no genera un conflicto directo entre el contraejemplo empírico descubierto y el sistema teórico original.

En consecuencia, es dable afirmar que la realidad social —el hecho- previo a su formalización teorética, constituye una entidad eminentemente dinámica, sin embargo, el proceso que intenta explicar o interpretar dicha dinámica, no alcanza a capturar todas las sutilezas empíricas y teóricas que la elaboración teórica contiene. El mundo es, sin duda, mucho más vertiginoso que el ritmo empírico del descubrimiento teórico. Las teorías sociales emergen por diferentes canales, sea a raíz de un problema de interés científico, o 
bien a partir de una relectura o interpretación de los constructos teóricos preexistentes. Sin mediar en su origen, las teorías nacen, poseen su momento de vigencia y, luego, se desgastan: efectivamente la realidad social puede, en principio, ser interpretada por cualquier tipo de teoría, no obstante las limitaciones naturales de la ciencia (y de cualquier tipo de conocimiento) respecto de la imposibilidad de lograr expresar toda la riqueza que un fenómeno pueda contener. Esta situación, si bien puede ser explicitada a través de una perspectiva historicista, relativa a la contextualización cultural de los significados y los conceptos, o bien ser interpretada a través de una teoría lingüística respecto del movimiento tropológico de las palabras; nosotros conjeturamos, por el contrario, que dicho proceso es esencialmente empírico, entendido éste como el desajuste de un nuevo hecho respecto de las teorías establecidas. En efecto, emergen nuevas prácticas sociales desde la óptica del sujeto- y nuevos escenarios sociales y políticos — desde el punto de vista de la estructura- pero estas nuevas dimensiones, a la larga, se hacen insostenibles si los sistemas conceptuales no se renuevan.

Esta descripción en lo formal es efectiva, pero en la práctica funciona muy lentamente: ello cristaliza en que una nueva teoría no elimina a una anterior. En las ciencias sociales la noción de teorías rivales no posee trascendencia epistemológica. Desde nuestra visión, las teorías no compiten entre sí y en principio, pese a que algunas posean sistemas conceptuales refutados empíricamente o anacrónicos respecto de la contextualización histórica, todas están en igualdad de condiciones para una operacionalización conceptual.

Entonces ¿En qué radica que algunas teorías estén de moda y otras estén en el limbo del olvido? La respuesta no está sujeta a parámetros historicistas ni a ningún tipo de explicación sumergida en factores externos a la misma ciencia, sino que el proceso de vigencia epistémica se centra, principalmente, en el acierto empírico que puede hacer en un momento específico, sea mediante una corroboración o bien por una refutación: el proceso interpretativo de ese hecho prolifera infinitamente, deriva en múltiples desenlaces conceptuales y significacionales, en nuevas teorías, estructuras relacionales entre hechos y sistemas de hipótesis. Si la proliferación teórica opera por un proceso de corroboración, éste supone el colapso simbólico y metafísico que la imaginación humana pueda soportar, pero ello bajo una intención optimista, en tanto que el objeto descubierto se extiende en su conformación teórica, cuestión que se traduce en investigaciones y literatura especializada. Muy por el contrario, si la proliferación teórica deviene por un acto de refutación, la generación teórica en torno al hecho refutado explosiona de forma negativa por cuanto la elaboración epistémica y literaria habrá de funcionar bajo los parámetros de una crítica epistemológica despiadada hacia la teoría clausurada.

B. Refutación Progresiva (Proceso de desgaste de la teoría). En principio, las teorías sociales no se eliminan entre si — suponiendo que la refutación empírica opere mecánicamente - como tampoco es la comunidad científica la que, deliberadamente, elimina a aquéllas que considera técnicamente obsoletas o fuera de contexto. Si bien este tipo de proceso nunca se lleva a efecto (como por ejemplo que algún crítico aportille 
sistemáticamente hasta la aniquilación las teorías de Durkheim o de Marx) sí ocurre que las teorías sociales se van superponiendo y sucediendo en el escenario epistémico: algunas quedan en desventajas y otras, claro está, adquieren mayor relevancia. Sin duda, ello genera un desgaste en los significados del sistema conceptual, aspecto que, sumado al acto re-interpretativo que la investigación realiza de las teorías disponibles termina por transformar el sentido de los modelos originales.

La superposición de modelos puede generar: a) Cambio conceptual en el cual cambia el referente y el significado, b) Cambio significacional en que se mantiene el referente lingüístico pero el significado cambia, o bien c) Cambio estético o formal en que cambia el referente pero los significados se mantienen. El proceso subsuntivo sobre el cual emerge una nueva teoría, o en su defecto, el proceso de refutación empírica - que funciona gradualmente- no genera cambios sustanciales en la visión global que una teoría posee respecto del mundo pero, no obstante ello, puede afectar espacios conceptuales de la teoría. Sin embargo, los significados y conceptos difícilmente se mantienen inalterados, por lo tanto, el desgaste de las palabras solo habrá de afectar la estructura teórica si ellos se repitiesen constantemente en diferentes ámbitos del sistema conceptual. Independiente de las contradicciones empíricas de las teorías y pese al cambio conceptual, los supuestos más generales sobre la realidad empírica se mantendrán medianamente estables o, por lo menos, en principio seguirán siendo utilizados.

Pese a que la teoría es objeto de crítica, tanto empírica como conceptual, muy remotamente habrá de ser eliminada como referente explicativo o interpretativo. En efecto, es posible que la estructura argumental eje de una teoría quede obsoleta, pero es muy probable que muchos de sus principios conceptuales por nimios que sean, queden operativos en el escenario epistémico: no por cuestiones contextuales sino porque la heterogeneidad interna de las teorías sociales es tan amplia y analítica que, muy probablemente, algún concepto teórico podrá sobrepasar la refutación empírica. Así ocurre básicamente con las teorías más clásicas de la sociología, las que, si bien han sido criticadas sistemáticamente, aún mantienen espacios teóricos, conceptos, perspectivas y orientaciones susceptibles de seguir siendo utilizadas, re-conceptualizadas o transformadas. Nos referimos, por ejemplo, a teorías como las de Siemmel, Comte o Durkheim, en tanto constituyen líneas teóricas que siguen marcando algún punto de referencia. En estos casos, las teorías, sólo en parte, son eliminadas del escenario científico, lo que se podría interpretar como un proceso efectivo de cambio epistémico, aunque en la práctica investigativa, en la literatura especializada y en las salas de clases se siga usufructuando de ellas. Pero ello, transcurrido más de un siglo de vigencia teórica, siempre y cuando la comunidad sociológica acepte la refutación total de estas teorías. Como eso difícilmente habrá de ocurrir, es muy probable que sólo algunas fracciones teóricas (dentro de un modelo) queden eliminadas. Bajo estos parámetros es posible plantear una refutación empíricamente débil (progresiva), pero certera respecto de los cambios conceptuales y de significados que pueda generar. Por ejemplo, las 244 
variables utilizadas por Durkheim en el trabajo sobre el suicidio hoy son totalmente obviadas, sin embargo su conceptualización respecto de la anomía aún es un referente conceptual que mantiene toda su vigencia en teorías criminológicas. Lógicamente, este mismo proceso es posible de ser extrapolado a teorías más contemporáneas, pero con la salvedad que éstas aún mantienen vigente el sentido e inspiración explicativa de la estructura teórica original, pese a que sus componentes conceptuales no contengan toda la fuerza explicativa y la correspondencia empírica que en la germinación de la teoría si poseían, como es el caso de la Teoría de la Dependencia. Ciertamente que, más allá de su acierto empírico, la teoría posee un espíritu sobre el cual se edifica la argumentación teórica. En estos casos, podemos apelar a que el espíritu de la teoría de la dependencia, su actitud crítica, su sentido argumental, su perspectiva frente a la realidad, posee aún asertividad epistémica. $^{12}$

Si bien las teorías sociales no son refutadas por las contradicciones empíricas que se puedan presentar, como tampoco es usual que se incorpore, explícita y concientemente, algún procedimiento de hipótesis ad hoc o que opere algún sistema formal de metodologías o estratagemas convencionalistas, aquí se apela por un proceso gradual y analítico de eliminación parcial de teorías sociales. Cuando se presentan desequilibrios y refutaciones evidentes - indistintamente del tipo de refutación, del tipo de teoría e independientemente de los diferentes contextos socioculturales donde ello ocurre - tales refutaciones funcionan lentamente y en diferentes estructuras de la teoría. La refutación progresiva, opera como un proceso histórico de desajustes técnicos, es decir, según la aplicabilidad contextual de la teoría, sus estructuras técnicas van desarrollando procesos de desmontaje y desequilibrio interpretativo. La crítica, en este sentido, es esencialmente interna, no es inmediata ni constante; su mecánica no está sujeta ni a contextos de moda ni a caprichos subjetivos: la falsación como proceso microtécnico de la refutabilidad puede proceder en enunciados empíricos, mientras que la refutabilidad, propiamente tal, bien puede operar en todo el sistema de hipótesis y a partir de ese espacio, desajustar sistemas lógicos, conceptos núcleos y definiciones. De igual forma, una refutación empírica respecto de un hecho concreto puede desmantelar sólo los enunciados relativos a la prescripción de ese hecho, pero con el tiempo puede hacer variar el significado del concepto sostén de aquel enunciado y culminar en el

\footnotetext{
${ }^{12}$ Más allá de su espíritu, las teorías constituyen compuestos epistémicos distribuidos en una serie de nichos técnicos sobre los cuales se edifica su sentido explicativo o interpretativo: cuestiones conceptuales, observacionales, de significación, de estructuración lógica, de sentido, de base empírica, una construcción específica del explicandum y un sistema general de hipótesis. Asimismo, existe una compenetración de orientaciones y perspectivas — respecto de intereses epistémicos, políticos y personales - entre la estructura original de una teoría (independientemente del trasfondo subjetivo y cultural de su creador) y la perspectiva que un investigador posterior le pueda imprimir en una investigación concreta; en esta compenetración funcionan tanto elementos técnicos (referidos al sistema teórico y observacional que implica la construcción del hecho; el explicandum) y elementos psicosociales supeditados a la contextualización histórica de la investigación.
} 
cambio radical del sentido del referente. En términos generales, la refutación progresiva opera desde las estructuras más simples de una teoría hasta culminar en desajustes estructurales de sentido epistémico, naturaleza ontológica y visión de realidad.

Vista desde Popper, la refutación progresiva opera en función de un contraejemplo empírico, o en su efecto, por el descubrimiento de un hecho divergente y opuesto a lo prescrito por un sistema de hipótesis formalizado. En el marco de la crítica interna, la refutación, procede sobre la unidad básica de una teoría: el contraejemplo actúa directamente sobre la base empírica y/o en un enunciado básico, que dentro del modelo de Popper, implica no sólo una acción nominal, sino que trasciende hacia la significación que el enunciado guarda respecto del hecho empírico o el proceso observacional que dice cristalizar. En este sentido, la refutación del enunciado básico conduce la edificación de la crítica epistemológica desde la estructura interna de la teoría, proceso que lo desarrolla en base al error empírico que el enunciado prescribió y no cumplió; y por el soporte significacional al que ese error conduce, el cual ya no se condice con la formulación conceptual original. Si bien la refutación puede constituir una operación epistemológica exitosa, en las ciencias sociales sólo actúa dentro de un contexto investigativo específico que no implica, por ejemplo, que los resultados de dicha investigación estén en un completo error, o que la teoría utilizada tenga que ser eliminada del escenario científico. El error del enunciado no implica una refutación directa de la teoría, ni un cambio conceptual automático: como se mencionó, el enunciado refutado - un punto específico dentro de la estructura interna de la teoría- actúa lenta y subrepticiamente, en razón de dos consecuencias: la primera, el enunciado supuestamente refutado no es eliminado, tampoco la teoría a la cual pertenece. Segundo, el enunciado no cambia de significado, como tampoco se incluye un nuevo referente para explicar el nuevo hecho. El enunciado (y con él la teoría) extiende su campo significacional y su capacidad interpretativa, pero en base a la información proporcionada por la investigación empírica: a él se vendría incorporar un nuevo matiz de estructuras significacionales adicionales no previstas.

Con todo, para las ciencias sociales, a diferencia de las ciencias naturales, el desajuste entre un enunciado y la conjetura teórica planteada —el sistema de hipótesis formalizado o la teoría propiamente tal - no se ve radicalmente afectada; el desequilibrio interno que podría generar un contraejemplo empírico - relativo a la correspondencia cognoscitiva entre teoría y hecho en razón de un sustrato de verdad (o error) del enunciado y del grado de conocimiento objetivo que el enunciado podría contener - no conduce directamente a una refutación teórica. En este sentido, el enunciado refutado $-\mathrm{y}$ con ello la posible contradicción generada al interior de la teoría- puede ser efectiva y objetiva, pero no genera un desajuste sustancial en la estructura general de la teoría. Traspasada la refutación del enunciado, las teorías siguen funcionando, siguen explicando o interpretando la realidad social (independientemente de lo que podría argumentar un defensor del historicismo, 246 
respecto de que una contradicción empírica puede ser objetiva en un contexto sociohistórico determinado, mientras que en otro contexto ni siquiera podría existir). En este sentido, si bien la refutación pudo ser certera, las teorías mantienen su vigencia epistémica y, ello, dado que la estructura de verdad de la teoría, respecto de los enunciados no refutados, más allá de seguir funcionando, siguen manteniendo cierto grado de certeza y de objetividad, lo que se condice con la extensión de significados a los que la teoría social puede apelar en caso de refutación. $^{13}$

Los desajustes empíricos y las refutaciones que se puedan desarrollar, responden a conceptos observacionales o enunciados, más que a conceptos teóricos: los conceptos observacionales corresponden a la elaboración lingüística que solo tiene lugar en la investigación específica, son constructos teóricos especializados que sólo dan cuenta de la realidad específica que se desea conocer. Los conceptos teóricos, a diferencia de los primeros, se construyen a partir de un sistema fundacional, funcionan como guías epistémicas, no poseen correspondencia empírica directa, pero conducen a la elaboración de conceptos especializados y/o observacionales. La refutación progresiva funciona en un marco investigativo y su acto de crítica apunta, precisamente, hacia los conceptos observacionales. Sin embargo la refutación de un concepto observacional o enunciado básico no es suficiente para eliminar la teoría; ello puesto que una investigación social difícilmente tendrá pretensiones de aplicar un modelo inductivo. Aunque la refutación fuese efectiva, el enunciado podrá seguir extendiendo su capacidad significacional; la investigación podrá ser exitosa y dar cuenta del problema planteado y la estructura teórica que sirve de base podrá darse por salvada. Sin embargo, es muy posible que, en investigaciones sucesivas, aquel enunciado manifieste desajustes con la realidad que pretende explicar, no precisamente bajo los supuestos de la falsabilidad y refutación empírica, sino por su omisión en los procesos investigativos

\footnotetext{
${ }^{13}$ Por ejemplo, la visión clásica de la Teoría de la Identidad suponía que muchas de las prácticas sociales de etnias indígenas se basaban en cánones de prácticas culturales afectivas, comunitarias y recíprocas (o en su efecto, en prácticas que desde el prejuicio occidental, son claramente irracionales). Sin embargo, hoy, la conceptualización de, por ejemplo, prácticas de intercambio comercial se sustentan en un evidente supuesto de racionalidad instrumental (sin cuestionar el sentido hermenéutico de la palabra "racionalidad instrumental"). Lo anterior implica argüir en dos frentes: a) Descartar de plano que las prácticas sociales indígenas se sustentan solamente en relaciones afectivas-comunitarias, b) El concepto de identidad (indígena), dado su dinamismo, podría admitir una serie de prácticas socio-culturales. En efecto, el concepto de identidad cultural no es ajeno a las prácticas indígenas basadas en relaciones occidentales de intercambio comercial. Otro ejemplo dice relación con el uso de la lengua: una visión radical de identidad étnica concibe que el patrón de identidad entre el sujeto y su historia cultural se edifica a partir del uso de la lengua materna. Sin embrago, muchas etnias desconocen totalmente su lengua de origen y ello no las hace menos indígenas o menos naturales. Ciertamente que la identidad lingüística, si bien es un factor relevante en procesos reivindicativos, es un elemento más dentro de un análisis identitario más complejo.
} 
que se lleven a efecto: una vez que un concepto teórico demuestre su error o falta de correspondencia empírica, difícilmente será utilizado en nuevas investigaciones o en nuevas elaboraciones de conceptos observacionales. Este hecho no elimina la teoría, como tampoco al enunciado, pero con el tiempo, se corre el riesgo de que muchos postulados de la teoría, muchos de sus sistemas de hipótesis queden desechados por la práctica científica.

No obstante lo anterior, los desajustes sucesivos, los constantes procesos de reformulación conceptual y la subsiguiente omisión por utilizar una teoría, habrán de gatillar un desequilibrio en su eje argumental: su sentido epistémico, su espíritu, su estructura ontológica respecto del mundo y el sujeto (independientemente de las bases filosóficas sobre las cuales es dable obtener un apoyo epistémico sustancial) verán lentamente mermadas sus pretensiones de interpretación y de explicación por cuanto los desajustes técnicos de la teoría no se condicen con las actuales pautas fácticas del proceso social, cultural y económico. Así, un proceso de refutación progresiva puede desestabilizar la estructura que demanda por la construcción del objeto científico y el desarrollo teórico y metodológico asociado. En efecto, cuando el sentido de la teoría difiere explícitamente de las condiciones empíricas de la realidad, se puede plantear en forma casi concluyente que la teoría ha sido definitivamente refutada. En este plano podemos plantear abiertamente que aquella teoría no se corresponde con cuestiones teóricas fundacionales (el explanans) y con su sentido cognoscitivo y axiológico original; en función de ese desajuste es posible argüir, por lo tanto, a favor de un proceso gradual de inflexión teórica y epistémica.

C. Proliferación de nuevas teorías a partir de la interpretación de los desajustes empíricos. El desgaste estructural de las teorías conlleva, con el tiempo, a su desventaja práctica, cada vez las teorías son menos utilizadas como marcos referenciales $\mathrm{y}$, en rigor, sólo comienza a usufructuarse parcialmente de ellas: algunos conceptos, algunas relaciones empíricas, fracciones del explanans o algún supuesto filosófico es reciclado ante la inminente clausura. El proceso de refutación progresiva ha implicado que muchos modelos teóricos no sean utilizados tal cual fueron originalmente diseñados, si bien no se eliminan ni se desechan del todo, sufren de lo que podríamos denominar, una refutación parcial que implica una crítica a fracciones o nichos conceptuales, según las incompatibilidades subsuntivas que la teoría va demostrando a través del tiempo. Pongamos por caso la Teoría Funcionalista que difícilmente podría ser aplicada en su forma pura en una investigación contemporánea; no porque esté pasada de moda o fuera del contexto epistémico actual, sino porque sólo muy forzadamente podríamos identificar estructuras sociales cuya existencia cultural se ajuste a la noción de funcionalidad. Si bien puede haber estructuras e instituciones sociales que, aparentemente, podrían tener un comportamiento funcional respecto de otras estructuras y/o instituciones, la generalidad del enunciado es demasiado imperativo y homogéneo como para conceptualizar a toda la realidad social bajo dicha óptica. En este sentido es posible extrapolar ciertas nociones teóricas de la funcionalidad y traducirlas, por 248 
ejemplo, bajo la perspectiva del proceso histórico que detalla el desarrollo o recorrido de un sistema o institución social, pero no bajo la noción de funcionalidad, sino bajo la noción de interrelaciones socioculturales. El desajuste empírico implica un desgaste a nivel de significados, esto es, clausurarlo ante la irrupción del contraejemplo empírico. Sin embargo, como se anotó anteriormente, la refutación no afecta al sentido general de la teoría la que, en principio, puede seguir aportando con otras esferas conceptuales: por ejemplo, en el caso del funcionalismo, el caso que existan procesos sociales (no sólo estructuras funcionales o disfuncionales) que actúan en forma explícita y otros en forma latente. $^{14}$

Bajo esta perspectiva, la refutación progresiva y parcializada conduce dentro del proceso subsuntivo a dos procesos epistémicos relativamente nuevos para el análisis epistemológico de la teoría social: a) La variación del significado teórico (Newton-Smith, 1987:173-179) y b) Un híbrido teórico y conceptual. Ambos procesos devienen en lo que podríamos rotular como la creación de nuevas teorías, que en el caso de las ciencias sociales no implica la eliminación irrestricta de la teoría rival, sino que se traduce en la superposición de diferentes modelos que bien se pueden compatibilizar con estructuras epistémicas existentes, o bien pueden ser totalmente opuestas. Si ocurriera esto último, la oposición entre dos teorías no sería bajo la noción de rivalidad sino, más bien, bajo la noción de disciplinas diferentes (aunque sus objetos sean los mismos), pero con una cierta complementariedad en los diseños teóricos utilizados. Cabe notar que este último aspecto no determina una atmósfera relativista — respecto de estructuras de verdad y objetividad — porque en el acto de superposición teórica es el criterio técnico de la refutabilidad el que determina qué teorías son más certeras, más objetivas y cuales nos acercan más a la verdad. C.1. La variación de significado: Los conceptos en el traspaso desde una teoría a otra debieran tener tres tipos de comportamiento: a) El significado de un concepto se mantiene invariante respecto de su origen teórico, independiente de la utilización posterior que se haga de ese concepto e independiente del modelo teórico en que es utilizado; b) El significado y el sentido de un concepto cambia al quedar totalmente refutada una teoría y c) El significado y el sentido del concepto cambia cuando este es extrapolado desde un modelo teórico a otro, independientemente de la situación epistemológica de la teoría de origen. Suponemos que con independencia de las situaciones a las cuales puedan estar efectos los conceptos, el proceso de movilidad conceptual siempre se remite a algún tipo de proceso de refutación empírica o de algún proceso de contraejemplo. En términos generales, el cambio teórico puede

\footnotetext{
${ }^{14}$ Independientemente de los múltiples ejemplos que aquí podrían detallarse, lo que se desea consignar es que las teorías se van replegando en sus aspectos explicativos e interpretativos, o en su efecto, la investigación empírica relega algunos de los postulados conceptuales de las teorías, mientras que otros son extrapolados a los requisitos que nuevas investigaciones demanden.
} 
tener su origen en un desajuste empírico a nivel del concepto observacional o, bien, un desajuste empírico referido al sentido epistémico de toda la teoría:

a) El significado de un concepto se mantiene invariante respecto de su origen teórico, con independencia de la utilización posterior que se haga de él e independientemente del modelo teórico en que es utilizado: los conceptos pueden actuar con autonomía de su origen teorético, es decir, sin importar las condiciones epistémicas de la teoría base, se impone por su grado de correspondencia empírica y explicativa. En estos casos, si bien no hablamos de una refutación propiamente tal, sí hablamos del poder empírico del concepto, de su capacidad interpretativa, de su temple y de su adecuación en diferentes modelos teóricos. Cuando un mismo concepto (con los matices significacionales que vengan al caso) es utilizado por diferentes teorías, la capacidad explicativa e interpretativa del concepto no radica en su simple significación, sino en el sentido general de la teoría en la cual ha sido utilizado. En estos casos, lo más probable es que el concepto utilizado no sea un referente teórico principal dentro de las teorías en cuestión; puede ser, que sólo en su teoría de origen sea un referente central pero no necesariamente en la segunda teoría, o bien, en ambas, sea un concepto más bien residual o de segundo orden. Por el contrario, si entre dos teorías el mismo concepto es un concepto eje de la estructura general del modelo lo más probable es que entre las dos teorías no existan grandes diferencias epistémicas y es muy factible que una sea el derivado de la otra. Si en una y otra teoría el significado del concepto es invariante, su diferenciación epistémica radicará en las relaciones conceptuales y teoréticas que al interior de la teoría se den. En estos casos no existe un cambio de significante, aunque si un cambio de sentido.

b) El significado y el sentido de un concepto cambia al quedar totalmente refutada la teoría. Cuando el concepto es totalmente dependiente del sistema teórico y al generarse un cambio teórico —o en su efecto un proceso de refutación empírica- el significado del concepto se verá alterado. Ello por dos razones: primero, la estructura significacional del concepto posee una estrecha vinculación con el sistema subsuntivo de la teoría; en otras palabras el concepto actúa como un concepto observacional que sólo adquiere sentido mediante la investigación empírica; si el concepto no encuentra una correspondencia directa verá su significación alterada, o en su efecto, el contenido del concepto no será relevante para el proceso interpretativo: el concepto puede darse por refutado. En estos casos hablamos más de un enunciado que de un concepto teórico. Segundo, el concepto actúa como eje epistémico dentro de la teoría: si la teoría es refutada en sus aspectos más sustanciales, inevitablemente el concepto verá alterado su significante, por cuanto el concepto constituye un aspecto explicativo fundamental dentro de la teoría, independientemente de que el concepto no haya sido directamente refutado. De igual forma si el concepto es extrapolado a un nuevo sistema teórico, cuyas referencias epistémicas no se condicen con la teoría original, su sentido puede variar respecto de la significación original que poseía. 
c) El significado del concepto siempre cambia al ser éste extrapolado desde un modelo teórico a otro, independiente de la situación epistemológica de la teoría de origen. Las teorías poseen una cierta referencia a cuestiones axiológicas y metodológicas; si las teorías van cambiando a través del tiempo y se van desarrollando nuevos modelos explicativos e interpretativos, el significado de los conceptos difícilmente permanecerá inmutable. El simple hecho de la creación teórica implica, en el plano del sentido significacional, una alteración respecto de su origen epistémico. En tales casos, los conceptos no son medidos por el contenido del referente, como tampoco por el contenido tradicional que puedan tener, sino que su poder explicativo radica en el acto hermenéutico que se pueda desarrollar sobre ellos. Si el eje epistémico de una nueva teoría, si su perspectiva y orientación difieren de la teoría original, el concepto verá transmutada no sólo su aplicabilidad empírica sino, también, el sentido de su significación. Conforme a esta visión, es el científico quien imprime un nuevo sentido y es él quien establece, por lo tanto, como usar los conceptos. En el lenguaje de NewtonSmith, a este último aspecto del proceso de cambio teórico se le denomina Variación Radical de Significado (1987:170ss.).

C.2. El híbrido teórico-conceptual. Al respecto, constatamos el hecho que muchas de las actuales teorías sociales se arman a partir de una serie de autores, conceptos y corrientes, de modo tal que, en el rigor epistémico, las investigaciones especializadas se constituyen como un híbrido conceptual. Las teorías actuales no poseen un eje teórico sustancial, por cuanto los grandes modelos en su intento holístico-deductivo derivan en exclusión empírica, esto es, por una parte intentar explicarlo todo, pero por la otra, excluyen los fenómenos, hechos o acciones que no encajan con sus postulados teóricos. Lo anterior conduce a que los investigaciones especializadas utilicen, más bien, una serie de perspectivas teóricas y conceptuales - sin importar si los términos conceptuales entre una y otra teoría son compatibles, contradictorios o divergentes - antes que sólo remitirse a un único modelo teórico. Lo que prevalece en este tipo de prácticas científicas no es el desarrollo teórico o la critica epistemológica; por el contrario, lo que importa desde la óptica del desarrollo técnico de la ciencia, es cómo se aprehende de la mejor manera posible el fenómeno o hecho que se desea estudiar; en estos casos funciona la máxima "mientras más, mejor", es decir mientras más autores, más teorías y más conceptos sea dable utilizar, mejor es el conocimiento que se puede extraer del fenómeno estudiado.

El desarrollo del híbrido teórico, en buena parte, se funda en la imposibilidad de conceptualizar una verdad teórica omnipresente. En este sentido la verdad del desarrollo científico radica en el hecho empírico, en su existencia observacional más que en la interpretación unidireccional en una única teoría. Para estos efectos, el concepto de teoría funciona como una estructura explicativa o interpretativa remitida exclusivamente al fenómeno que se desea estudiar. Así, las relaciones explicativas, conceptuales y significacionales son específicas y están determinadas temporal y espacialmente según los intereses que cada investigación e investigador pone como criterio técnico. Esta 
práctica puede constituir una especie de metodología investigativa, es decir la construcción teórica debe necesariamente compilarse según dos criterios: a) Las teorías que objetivamente sirvan para la investigación, según otras aplicaciones investigativas, b) Las teorías de moda que, sin importar el sentido epistémico por el cual fueron concebidas, en virtud de su aplicabilidad son susceptibles de ser extrapoladas a una investigación empírica.

En el marco de lo expuesto, la investigación científica no pretende poner a prueba una teoría, por lo menos bajo lo que Popper entiende por "poner a prueba" (1994:61-179), como tampoco intenta desarrollar algún nuevo concepto o teoría. Básicamente el híbrido conceptual es una instrumentalización de los referentes teóricos disponibles que no provoca ni conduce procesos de inflexión epistémica, aunque si promueve la acumulación de conocimiento empírico, o nuevas conceptualizaciones a partir de nuevas experiencias, lo que deriva en dos cuestionamientos: primeramente ¿Cómo se genera refutación progresiva bajo el marco de un híbrido teórico? y segundo ¿Bajo qué supuestos es dable la generación nuevas teorías (y no sólo nuevas investigaciones)?

En las investigaciones híbridas se generan desajustes empíricos pero sólo funcionan a nivel del concepto utilizado, mientras que la lógica y el sentido general de la investigación pueden, en principio, seguir funcionando hasta que otra estructura teórica diga lo contrario, independientemente de que ella utilice los mismos conceptos que la investigación rival. Así, en el marco de una investigación especializada, la refutación progresiva y parcializada sobre los enunciados y conceptos viene operando según el uso a que están sujetos y funciona desde el momento mismo en que una teoría entra al juego disciplinario-práctico, aunque no se desarrollen acciones directas, concretas y deliberadas para refutar dichas teorías.

\section{CONCLUSIÓN}

En términos generales, las teorías sociales efectivamente son refutadas a través del tiempo, a partir del equilibrio entre teoría, perspectiva y elementos empíricos. Sin duda que el cambio teórico se habrá de generar a partir de un acto de crítica ante los híbridos conceptuales y ello en función de las limitantes interpretativas que el híbrido desarrolla en una teoría. Para rescatar la creación teórica habría que desarrollar, en cada desajuste empírico, una relectura de los explanans con los cuales las teorías trabajan, en razón de establecer conexiones teóricas audaces, independientemente de las correlaciones empíricas que se puedan, a priori, establecer. La creación de nuevas teorías, muy al estilo de Popper, debieran incluir serios intentos por re-interpretar la realidad social y la utilización de los fundamentos epistémicos a los que las teorías se ven supeditados, más que reproducir acríticamente los clásicos, las investigaciones consabidas y los mismos conceptos y perspectivas de siempre. Desde nuestra perspectiva, las nuevas teorías y el proceso de inflexión epistémica que debiera ocurrir junto con la proliferación de conocimiento nuevo, es un proceso 
empírico que no se basa directamente en la simple observación, sino que consiste en una estructura epistémica que debiera tener, a lo menos, las siguientes consideraciones: a) muy en la senda de Popper, la elaboración de nuevas teorías requiere de un proceso hipótetico-deductivo, más que de un proceso observacional directo, b) este proceso habrá de fundamentar su elaboración conceptual en las relaciones empíricas que son posibles de extraer de algún explanans o fundamento filosófico, c) las nuevas elaboraciones conceptuales y teóricas y las relaciones lógicas posibles de generar nuevas perspectivas y nuevas leyes de subsunción, necesitan una serie de contrastaciones empíricas que se irán formulando en las aplicaciones investigativas que realiza la ciencia aplicada y especializada. Éstas entran en un proceso continuo e histórico que va desechando conceptos, teorías y perspectivas que, a su vez, incentivan la generación de nuevos modelos interpretativos, que según nuestra apreciación deambulan entre a) la mera reproducción y extrapolación de teorías y b) en la generación de los híbridos teórico-conceptuales; ambos dirigidos a solucionar problemas de interés. Conforme a estos supuestos, la inflexión teórica y epistémica en las ciencias sociales está inmersa en un horizonte de historicidad que implica un desarrollo empírico e interpretativo, progresivo y parcializado según las diferentes aplicaciones técnicas que un investigador establece respecto de los componentes teóricos y conceptuales que son posibles de extrapolar y combinar de las diferentes teorías existentes, cuyo uso, en principio, no habrá de radicar en la valoración subjetiva y contextual que por sobre dicha teoría se promulgue. En tal sentido, apelamos a que el desarrollo de la teoría es un proceso que utiliza, muy a pesar de las posturas relativistas, una serie de criterios racionales y técnicos, referidos precisamente a la complejidad y feracidad que las nuevas elaboraciones teóricas pueden tener respecto de un problema de interés científico. No negamos que el proceso general de proliferación de nuevas teorías se dé bajo una realidad histórica concreta pero, desde nuestra perspectiva, el análisis epistemológico que sólo busca el desarrollo teórico a través de la contextualización espacio-temporal, no podrá dar cuenta que la generación de conocimiento teórico se edifica — más allá de criterios empíricos y observacionales - a partir de una especial hermenéutica empirio-historicista que habrá de sustentar lo que aquí hemos denominado Refutación Progresiva.

Universidad Arturo Prat* Instituto de Estudios Andinos Isluga Avda. Arturo Prat 2120. Iquique (Chile) cristian.ortega@unap.cl 
BIBLIOGRAFÍA

Berger, P.; Luckmann, T. La construcción social de la realidad. Buenos Aires: Amorrortu, 2001.

Feyerabend, Paul. Contra el método: Esquema de una teoría anarquista del conocimiento. Buenos Aires: Orbis, S. A., 1984.

Giddens, Anthony. Las nuevas reglas del método sociológico. Buenos Aires: Amorrortu, 1997.

Kuhn, Thomas. La Tensión Esencial. México: F.C.E., 1996.

Lakatos, Imre. Escritos Filosóficos. La metodología de los programas de investigación científica. Madrid: Alianza, 2002.

Historia de las ciencias y sus reconstrucciones racionales. Madrid: Tecnos, 1993.

Newton-Smith, W. H. La racionalidad de la ciencia. Barcelona: Paidós, 1987.

Popper, Karl. La lógica de la investigación científica. Madrid: Tecnos, 2004.

------ Los dos problemas fundamentales de la epistemología. Madrid: Tecnos, 1998.

------ Conjeturas y refutaciones: El desarrollo del conocimiento científico. Barcelona: Paidós, 1994.

------ La miseria del historicismo. Madrid: Alianza, 1973.

Schutz, Alfred. El problema de la realidad social. Buenos Aires: Amorrortu, 1995.

Sokal, Alan; Bricmont, Jean. Imposturas Intelectuales. Barcelona: Paidós, 1999. 